\title{
ASYMPTOTIC SOLUTION WITH RESPECT TO A PARAMETER OF A DIFFERENTIAL EQUATION HAVING AN IRREGULAR SINGULAR POINT
}

\author{
NICHOLAS D. KAZARINOFF ${ }^{1}$
}

1. Introduction. The subject of this note is the asymptotic solution, for large absolute values of a complex parameter $\lambda$, of the differential equation of the type

$$
\left(z-z_{0}\right)^{\nu} \frac{d^{2} w}{d z^{2}}+\lambda\left(z-z_{0}\right)^{\nu / 2} P_{1}(z, \lambda) \frac{d w}{d z}+\lambda^{2} P_{2}(z, \lambda) w=0,
$$

with $\nu-2$ a positive real constant. The complex variable $z$ is to be confined to a bounded neighborhood of the irregular singular point $z_{0}$. The analogous problem for a class of $n$th order differential equations, containing (1) for integral $\nu \geqq 6$, has been discussed by Hurd [2]. His analysis is somewhat complicated, and it appears that a clearer and more direct investigation of the asymptotic solutions of (1) than is attainable by the methods applied in [2] is possible. This is accomplished largely by adapting the formal structure of the analysis [3] used in the discussion of (1) for $\nu=2$.

The case $\nu>2$ discussed here resembles, in some respects, that when $\nu<2$. In order to contrast the case $\nu<2$ with the present one, we first outline the nature of our results.

Suitable changes of the variables in (1) reduce it to the form

$$
L(u)=0, \quad L=d^{2} / d x^{2}-\lambda^{2} Q(x, \lambda) .
$$

By way of hypothesis it is to be assumed that the function $x^{\nu} Q(x, \lambda)$ is single-valued and analytic in $x$ throughout a bounded region $R$ containing the origin and in $\lambda$ at infinity. The important restriction is made that $x^{\nu} Q(x, \infty)$ is nonzero in $R$ with $\lambda$ chosen so that $\left.x^{\nu} Q(x, \infty)\right|_{x=0}=1$. A further hypothesis upon $R$ is stated in $\S 3$.

Let $n$ be any non-negative integer. It will be shown that a function $\xi(x, \lambda)$ may be defined and a pair of polynomials in $1 / \lambda$,

$$
A_{ \pm n}(x, \lambda)=\sum_{0}^{n} \frac{\alpha_{ \pm j}(x)}{\lambda^{i}}
$$

Presented to the Society, December 29, 1954 ; received by the editors July 16, 1954 and, in revised form, March 21, 1955.

1 This paper was written in the course of research conducted under the sponsorship of the Office of Naval Research at the University of Wisconsin. 
determined such that the expressions

$$
y_{ \pm n}(x, \lambda)=e^{ \pm \xi} A_{ \pm n}(x, \lambda)
$$

approximate solutions of (2) with an error of $e^{ \pm \xi} O\left(\lambda^{-n-1}\right) .{ }^{2}$ The functions $\alpha_{ \pm j}(x)$ are determined by means of a simple algorithm so that the results given here are more explicit than those of [2]. This algorithm is essentially the same as that used by Birkhoff in 1908 [1] in the treatment of (1) for $\nu=0$. The decisive difference is in the choice of $\xi(x, \lambda)$. Moreover, if one were to determine the asymptotic representations of the solutions of (2) in accordance with the procedures developed by Birkhoff [1] and Langer [4], among others, for $\nu<2$ or by the analysis referred to in [3], the remainder terms multiplying $e^{ \pm \xi}$ would not remain bounded as $z \rightarrow z_{0}$. As is the case when $\nu=0$ or 2 , the asymptotic forms given here are made up of elementary functions. As is not the case when $\nu=0$ or 2 but as is the case in general when $\nu<2$, the Stokes' phenomenon appears; that is, no solution is asymptotic to one of the expressions $y_{ \pm n}(x, \lambda)$ everywhere in $R$. Previously, asymptotic forms approximating solutions of (2) to terms of order $e^{ \pm \xi} O\left(\lambda^{-n-1}\right)$ where $n$ is any non-negative integer have been found only for $\nu=-2,-1,0,1,2$. In this respect the theory to be given for $\nu>2$ is more complete than that known for $\nu<2$. But the fact that $z_{0}$ is an irregular singular point of (2) has prevented the author from obtaining connection formulas for the solutions. Such formulas are known if $\nu<2$ and are not necessary if $\nu=0$ or 2 . The problem of finding connection formulas in $\lambda$ for fixed $z$ has remained unsolved in all cases.

2. Definition of $\xi(x, \lambda)$. The hypotheses upon $Q(x, \lambda)$.guarantee that it may be expanded in the series ${ }^{3}$

$$
Q(x, \lambda)=\sum_{0}^{\infty} \frac{q_{j}(x)}{\lambda^{i}}, \quad \text { when } x \neq 0 \text { and }|\lambda|>N,
$$

where the functions $x^{\nu} q_{j}(x)$ are analytic in $R$. This series may be differentiated termwise with respect to $x$.

Let $\phi(x)$ be the square root of $q_{0}(x)$ such that

$$
\left.x^{\nu / 2} \phi(x)\right|_{x=0}=1 \text {. }
$$

A sequence of polynomials $\kappa_{j}^{*}(x), j=0,1, \cdots$, of degree $[1+\nu / 2]$ or less is determined by the conditions that the following limits exist

${ }^{2}$ The notation $O\left(\lambda^{-n}\right)$ is used to designate a bounded function of $x$ which when multiplied by $\lambda^{n}$ is a bounded function of $\lambda$ as well.

${ }^{3}$ The letter $N$ is to be used as a generic symbol for a positive constant. 
(if $2<\nu<4$, then $\kappa_{2}^{*}(x)$ will be the sum of a polynomial and a term $\left.c x^{\nu-2}\right)$ :

(a) $\lim x^{\nu / 2}\left[\kappa_{j}^{*}(x) q_{0}(x)-q_{j}(x)\right]=l_{j}, \quad j \neq 0,2$,

(b) $\kappa_{0}^{*}(x) \equiv 1$,

(c) $\lim _{x \rightarrow 0} x^{\nu / 2}\left[\kappa_{2}^{*}(x) q_{0}(x)-q_{2}(x)+\frac{\nu}{4}\left(\frac{\nu}{4}-1\right) x^{-2}\right]=l_{2}$,

wherein the $l_{j}$ are constants. Because of the analyticity of $x^{\nu} Q(x, \lambda)$ in $x$ and $\lambda$, it is readily seen that the function $\kappa^{2}(x, \lambda)-\kappa_{2}^{*}(x) / \lambda^{2}$, where

$$
\kappa^{2}(x, \lambda)=\sum_{0}^{\infty} \frac{\kappa_{j}^{*}(x)}{\lambda^{j}},
$$

is also analytic for $x$ in $R$ and $|\lambda|>N$. The root

$$
\kappa(x, \lambda)=\sum_{0}^{\infty} \frac{\kappa_{j}(x)}{\lambda^{j}}
$$

is chosen so that $\kappa_{0}(x) \equiv 1$. We now define $\xi(x, \lambda)$ to be any primitive of

$$
\xi^{\prime}(x, \lambda)=\lambda \kappa(x, \lambda) \phi(x) .
$$

Lastly, we introduce a function $\Theta(x, \lambda)$ through the relation

$$
\left[\xi^{\prime}(x, \lambda)\right]^{2}-\lambda^{2} Q(x, \lambda)=\lambda \Theta(x, \lambda)+\frac{\nu}{4}\left(1-\frac{\nu}{4}\right) x^{-2}
$$

Referring to the definitions (6) of $\kappa_{j}^{*}(x)$, it is evident that $x^{\nu / 2} \Theta(x, \lambda)$ is bounded for $|\lambda|<N$ and $x$ in $R$. Moreover,

$$
\Theta(x, \lambda)=\sum_{0}^{\infty} \frac{\theta_{j}(x)}{\lambda^{j}}, \quad x \neq 0 .
$$

3. The surfaces $R_{x}$ and $R_{\xi}$. For fixed $\lambda$ the function $\xi(x, \lambda)$ is in general multiple-valued in $R$. We therefore consider $R$ to be covered by a Riemann surface appropriate to a single-valued representation of $\xi(x, \lambda)$. This surface will be designated $R_{x}$. The Riemann surface over which the inverse function $\xi^{-1}(x, \lambda)$ is single-valued we denote $R_{\xi}$. The portions of the positive and negative axes of reals included on $R_{\xi}$ may be thought of as dividing this surface into regions $\boldsymbol{\Xi}_{j, k}$, $j=1,2, k=0, \pm 1, \pm 2, \cdots$, where

$$
\begin{array}{rlrl}
\Xi_{1, k}: & 2 k \pi & \leqq \arg (\xi) & \leqq 2(k+1) \pi, \\
\Xi_{2, k}: & (2 k-1) \pi & \leqq \arg (\xi) \leqq(2 k+1) \pi .
\end{array}
$$


The boundaries of the images of these regions upon $R_{x}$ are, of course, dependent upon the parameter $\lambda$. If the constant $\nu$ is rational, the surfaces $R_{x}$ and $R_{\xi}$ may be of finite order; and in this case, only a finite number of the regions $\boldsymbol{\Xi}_{j, k}$ will be distinct. On the other hand, if $\nu$ is irrational, the regions $\boldsymbol{\Xi}_{j, k}$ are distinct for all allowed $j$ and $k$.

A curve joining a point of $\boldsymbol{\Xi}_{j, k}$ to the point at infinity as approached along the ray arg $(\xi)=(2 k-j+2) \pi$ and along which $R(\xi)$ is monotonic will be called a $\Gamma$-curve. The image of such a curve upon $R_{x}$ will be called a $\gamma$-curve. The final assumption to be made upon the character of the region $R$ may now be stated. We assume that for the value of $\lambda$ under consideration and for each integer $k$ all points of $\Xi_{j, k}, j=1$ or 2, may be connected to $\xi=\infty$ by $\Gamma$-curves chosen so that

$$
\left|\int_{\gamma} d x\right|<N, \text { when } \xi(x, \lambda) \text { is in } \Xi_{j, k} .
$$

We note that inasmuch as $\xi$ is dependent upon $\lambda$, a single region $R$ may not satisfy the above hypothesis for all $\lambda$ with $|\lambda|>N$.

4. Determination of $A_{ \pm n}(x, \lambda)$. Direct computation shows that

$$
L\left(y_{ \pm n}\right)=e^{ \pm \xi} H_{ \pm n}
$$

where

$$
\begin{aligned}
H_{ \pm n}(x, \lambda)= & A_{ \pm n}^{\prime \prime}(x, \lambda) \pm 2 \xi^{\prime}(x, \lambda) A_{ \pm n}^{\prime}(x, \lambda) \\
& +\left[\left(\xi^{\prime}(x, \lambda)\right)^{2}-\lambda^{2} Q(x, \lambda) \pm \xi^{\prime \prime}(x, \lambda)\right] A_{ \pm n}(x, \lambda) .
\end{aligned}
$$

The function $\kappa(x, \lambda)$ has been so chosen that it is possible to determine the $\alpha_{ \pm j}(x)$ to be bounded functions by equating the first $n+1$ terms of the series in descending powers of $\lambda$ for $H_{ \pm n}(x, \lambda)$ to zero. These terms vanish provided that the $\alpha$ 's satisfy the following differential equations

$$
\begin{aligned}
& \pm 2 \phi \alpha_{ \pm 0}^{\prime}+\left(\theta_{0} \pm \phi^{\prime}\right) \alpha_{ \pm 0}=0 \\
& \pm 2 \phi \alpha_{ \pm 1}^{\prime}+\left(\theta_{0} \pm \phi^{\prime}\right) \alpha_{ \pm 1}+\alpha_{ \pm 0}^{\prime \prime}+(\nu / 4)(1-\nu / 4) x^{-2} \\
&+2 \kappa_{1} \phi \alpha_{ \pm 0}^{\prime}+\left[\theta_{1} \pm\left(\kappa_{1} \phi\right)^{\prime}\right] \alpha_{ \pm 0}=0 \\
& \pm 2 \phi \alpha_{ \pm m}^{\prime}+\left(\theta_{0} \pm \phi^{\prime}\right) \alpha_{ \pm m}+\alpha_{ \pm(m-1)}^{\prime \prime} \pm 2 \phi \sum_{1}^{m} \kappa_{j} \alpha_{ \pm(m-i)}^{\prime} \\
&+\sum_{1}^{m}\left[\theta_{i} \pm\left(\kappa_{j} \phi\right)^{\prime}\right] \alpha_{ \pm(m-j)}=0, \\
& m=2,3, \cdots, n .
\end{aligned}
$$


This is the case if

$$
\begin{aligned}
\alpha_{ \pm 0}(x)= & \phi^{-1 / 2} \exp \left(\mp \int_{0}^{x} \frac{\theta_{0}}{2 \phi} d t\right), \\
\alpha_{ \pm 1}(x)= & \mp \alpha_{ \pm 0}(x) \int_{0}^{x}\left\{ \pm\left[\kappa_{1} \theta_{0}+\kappa_{1}^{\prime} \phi\right]\right. \\
& \left.+\frac{\nu}{4}\left(1-\frac{\nu}{4}\right) x^{-2}+\theta_{1}+\frac{\alpha_{ \pm 0}^{\prime \prime}}{\alpha_{ \pm 0}}\right\} \frac{d t}{2 \phi}, \\
\alpha_{ \pm m}(x)= & -\alpha_{ \pm 0}(x) \int_{0}^{x}\left\{2 \phi \sum_{1}^{m} \kappa_{j} \alpha_{ \pm(m-j)}^{\prime} \quad, \quad m=2,3, \cdots, n .\right.
\end{aligned}
$$

The functions $x^{-v / 4} \alpha_{ \pm 0}$ are analytic in $R$, and the functions $x^{-v / 4-1} \alpha_{ \pm m}$, $m=1, \cdots, n$, are bounded in $R$. Consequently, $x^{\nu / 4} H_{ \pm n}(x, \lambda)$ is bounded in $R$.

The linear independence of $y_{+n}(x, \lambda)$ and $y_{-n}(x, \lambda)$ can now be established. The Wronskian of these functions will be denoted $W(x, \lambda)$, and it is easily seen from (4) that

$$
W(x, \lambda)=\left|\begin{array}{ll}
A_{+n} & A_{+n}^{\prime}+\xi^{\prime} A_{+n} \\
A_{-n} & A_{-n}^{\prime}-\xi^{\prime} A_{-n}
\end{array}\right| .
$$

At $x=0$ this relation becomes $W(0, \lambda)=-2 \lambda_{\kappa}(0, \lambda)$. It can be shown that $[3, \S 3]$

$$
W^{\prime}(x, \lambda)=\left(1 / \lambda^{n}\right)\left\{A_{+n} H_{-n}-A_{-n} H_{+n}\right\} .
$$

Integrating this expression from zero to $z$, we may conclude that

$$
W(x, \lambda)=-2 \lambda_{\kappa}(0, \lambda)+B(x, \lambda) / \lambda^{n}
$$

for $x$ in $R$ and $|\lambda|>N$, where $B(x, \lambda)$ designates a bounded function of $x$ and $\lambda$. Thus for $|\lambda|$ sufficiently large $W(x, \lambda)$ is nonzero in $R$, and therefore the functions $y_{ \pm n}(x, \lambda)$ are linearly independent.

5. Asymptotic solutions of $L(u)=0$. In order to show the asymptotic representation of solutions of $L(u)=0$ by the functions $y_{ \pm n}(x, \lambda)$, we compare $L(u)=0$ with the differential equation satisfied by the functions

$$
z_{ \pm n}(x, \lambda)=[\lambda / W(x, \lambda)]^{1 / 2} y_{ \pm n}(x, \lambda)
$$


This equation may be written in the form $[3, \S 3]$

$$
d^{2} z / d x^{2}-\left[\lambda^{2} Q(x, \lambda)-\Omega(x, \lambda) / \lambda^{n}\right] z=0,
$$

in which

$$
\begin{gathered}
\Omega(x, \lambda)=-\lambda^{n}\left[E / W+(F / 2 W)^{2}+(F / 2 W)^{\prime}\right], \\
F(x, \lambda)=\left|\begin{array}{ll}
A_{+n} & H_{+n} \\
A_{-n} & H_{-n}
\end{array}\right|, \quad \text { and } E(x, \lambda)=\left|\begin{array}{ll}
H_{+n} & A_{+n}^{\prime}+\xi^{\prime} A_{+n} \\
H_{-n} & A_{-n}^{\prime}-\xi^{\prime} A_{-n}
\end{array}\right| .
\end{gathered}
$$

The observations made in $\S 4$ upon the structure at $x=0$ and behavior in $R$ of the functions $\alpha_{ \pm j}(x), H_{ \pm n}(x, \lambda)$ and $W(x, \lambda)$ reveal that $x^{\nu / 2} \Omega(x, \lambda)$ is bounded in $R$. That $\Omega(x, \lambda)$ is bounded for $|\lambda|>N$ and each fixed $x \neq 0$ may be concluded from the behavior of these same functions at $\lambda=\infty$.

The relation

$$
\left|\begin{array}{cc}
z_{+n} & z_{+n}^{\prime} \\
z_{-n} & z_{-n}^{\prime}
\end{array}\right|=\frac{\lambda}{W}\left|\begin{array}{cc}
y_{+n} & y_{+n}^{\prime} \\
y_{-n} & y_{-n}^{\prime}
\end{array}\right|
$$

follows directly from (10). Consequently, the Wronskian of $z_{+n}(x, \lambda)$ and $z_{-n}(x, \lambda)$ is equal to $\lambda$. Thus for each integer $k$ a pair of linearly independent solutions, $u_{1, k}(x, \lambda)$ and $u_{2, k}(x, \lambda)$, of $L(u)=0$ is given by the formulas

$$
\begin{aligned}
& u_{j, k}(x, \lambda)=z_{ \pm n}(x, \lambda) \\
& -\frac{1}{\lambda^{n+1}} \int_{\gamma}\left[z_{+n}(x) z_{-n}(t)-z_{+n}(t) z_{-n}(x)\right] \Omega(t) u_{j, k}(t) d t \\
& \quad j=1,2, k=0, \pm 1, \cdots .
\end{aligned}
$$

In these and subsequent formulas the upper sign is to be used when $j=1$, the lower sign when $j=2$. As indicated the path of integration is to be a $\gamma$-curve.

With the aid of the abbreviations

$$
\begin{aligned}
Z_{ \pm n}= & \phi^{1 / 2} e^{\mp \xi} z_{ \pm n}, \quad U_{j, k}=\phi^{1 / 2} e^{\mp \xi} u_{j, k}, \\
K_{j}(x, t, \lambda)= & \pm \frac{\Omega(t)}{\phi(t)}\left[Z_{ \pm n}(x) Z_{\mp n}(t)-Z_{ \pm n}(t) Z_{\mp n}(x)\right. \\
& \cdot \exp (\mp 2[\xi(x)-\xi(t)])],
\end{aligned}
$$

equation (11) may be written as

$$
U_{j, k}(x)=Z_{ \pm n}(x)-\frac{1}{\lambda^{n+1}} \int_{\gamma} K_{j}(x, t, \lambda) U_{i, k}(t) d t .
$$


The functions $\Omega(x, \lambda) / \phi(x)$ and $Z_{ \pm n}(x, \lambda)$ are bounded on $R_{x}$ so that the kernel $K_{j}(x, t, \lambda)$ is a bounded function aside from the exponential factor. The $\gamma$-curves have been so chosen that this exponential is bounded along a $\gamma$-curve. Thus $K_{j}(x, t, \lambda)$ is bounded for $\xi$ in $\boldsymbol{\Xi}_{j, k}$.

Equation (13) is formally satisfied by the infinite series

$$
U_{j, k}(x)=Z_{ \pm n}(x)+\frac{1}{\lambda^{n}} \sum_{m=1}^{\infty} \frac{Z_{ \pm n}^{(m)}(x)}{\lambda^{m}}
$$

wherein

$$
Z_{ \pm n}^{(m)}(x)=\int_{\gamma} K_{j}(x, t, \lambda) Z_{ \pm n}^{(m-1)}(t) d t, \quad Z_{ \pm n}^{(0)}(x)=Z_{ \pm n}(x) .
$$

Let $M$ denote an upper bound of

$$
\left|K_{j}(x, t, \lambda)\right| \cdot\left|\int_{\gamma} d t\right|, \quad\left|Z_{ \pm n}(x)\right| \quad \text { for } \xi(x, \lambda) \text { in } \Xi_{i, k} ;
$$

then

$$
\left|Z_{ \pm n}^{(m)}(x)\right| \leqq M^{m+1} \quad \text { for } \xi(x, \lambda) \text { in } \Xi_{j, k} .
$$

This upper bound exists by virtue of the hypothesis made in $\$ 3$ and the remarks of the preceding paragraph. From (15) it is clear that the infinite series (14) converges uniformly when $|\lambda|>M$ and $\xi$ is in $\Xi_{j, k}$. Referring to the expressions (12), (10), and (4), equation (14) may now be rewritten in terms of $u_{j, k}(x, \lambda)$ and $y_{ \pm n}(x, \lambda)$. In this connection we note that

$$
[\lambda / W(x, \lambda)]^{1 / 2}=B(\lambda)+O\left(\lambda^{-n-1}\right),
$$

where $B(\lambda)$ is a bounded function of $\lambda$.

To summarize the conclusions of our discussion we state the following

THEOREM. Under the hypotheses stated in $\$ \$ 1$ and 3, over each region $\Xi_{j, k}$ on $R_{\xi}$ the given differential equation (2) has a solution $u_{j, k}(x, \lambda)$ of the form

$u_{j, k}(x, \lambda)=e^{ \pm \xi}\left[A_{ \pm n}(x, \lambda)+\frac{O\left(\lambda^{-n-1}\right)}{\phi^{1 / 2}}\right], \quad j=1,2, k=0, \pm 1, \cdots$.

The functions $\xi(x, \lambda), A_{ \pm n}(x, \lambda)$, and $\phi(x)$ and the region $\boldsymbol{\Xi}_{j, k}$ are described by the several formulas (3), (5), (7), (8), and (9). Since the regions $\Xi_{1, k}$ and $\Xi_{1, k-1}$ cover the region $\Xi_{2, k}$, the theorem determines a pair of linearly independent solutions of (2) for each $x \neq 0$ in $R_{x}$. 
The asymptotic representation of a solution $u_{j, k}(x, \lambda)$ given by the theorem does not in general hold over all of $R_{x}$ but only on the image of $\boldsymbol{\Xi}_{j, k}$.

\section{REFERENCES}

1. G. D. Birkhoff, On the asymptotic character of the solution of certain differential equations containing a parameter, Trans. Amer. Math. Soc. vol. 9 (1908) pp. 214-231.

2. C. C. Hurd, Asymptotic theory of linear differential equations singular in the variable of differentiation and in a parameter, Tôhoku Math. J. vol. 44 (1938) pp. 243274.

3. N. D. Kazarinoff and R. W. McKelvey, Asymptotic solution of differential equations in a domain containing a regular singular point, Canadian Journal of Mathematics vol. 8 (1956).

4. R. E. Langer, On the asymptotic solution of differential equations, with reference to the Stokes' phenomenon about a singular point, Trans. Amer. Math. Soc. vol. 37 (1935) pp. 397-416.

\section{Purdue University}

\section{ON STIELTJES INTEGRATION}

\section{E. J. MCSHANE}

Among the theorems concerning the Stieltjes integral there are two which are established for integrals in one-dimensional space, but not in spaces of more than one dimension. These are (I) if $\int f d g$ exists, $f$ and $g$ have no common discontinuity; (II) if $\int f d g$ exists, and $g$ is of bounded variation and $t$ is its total-variation function, then $\int f d t$ exists. The method of proof for one dimension ${ }^{1}$ does not extend to higher dimensions. In this note extensions of these theorems to $n$ dimensions are proved for the ordinary Stieltjes integral and for a modified form of it. ${ }^{2}$

1. Definitions. Throughout this note we shall assume that $f$ is real-valued and bounded on a set $D$ in the space $R^{n}$, and that $g$ is real-valued on $R^{n}$. For each interval $I \subset R^{n}$ we define $\Delta_{g} I$ in the usual way, as the sum of $2^{n}$ terms each of which is \pm 1 times the value of $g$ at a vertex of $I$. If $B$ is a closed interval contained in $D$, an extended partition of $B$ is a set $P=\left\{I_{1}, I_{2}, \cdots, I_{k}, x_{1}, x_{2}, \cdots, x_{k}\right\}$ in

Presented to the Society, April 16, 1955; received by the editors March 4, 1955.

${ }^{1}$ L. M. Graves, Theory of functions of real variables, McGraw-Hill, 1946, p. 263, Theorem 4, and p. 273, Theorem 14.

2 E. J. McShane and T. A. Botts, A modified Riemann-Stieltjes inlegral, Duke Math. J. vol. 19 (1952) p. 293. 